\title{
Ifosfamide-loaded poly (lactic-co-glycolic acid) PLGA-dextran polymeric nanoparticles to improve the antitumor efficacy in Osteosarcoma
}

\author{
Bin Chen ${ }^{1+}$, Jie-Zuan Yang ${ }^{2 \dagger}$, Li-Feng Wang ${ }^{1}$, Yi-Jun Zhang ${ }^{1}$ and Xiang-Jin Lin ${ }^{1 *}$
}

\begin{abstract}
Background: Osteosarcoma is a typical bone cancer that primarily affects adolescents. The therapeutic activity of drugs is limited by their severe drug-related toxicities, therefore, a therapeutic approach which is less toxic and highly effective in tumor is of utmost importance.

Method: In this study, ifosfamide-loaded poly (lactic-co-glycolic acid) (PLGA)-dextran polymeric nanoparticles (PD/IFS) was developed and studied its anticancer efficacy against multiple osteosarcoma cancer cells. The drug-loaded nanoparticle was characterized for physical and biological characterizations.

Results: The formulated PD/IFS showed a high drug loading capacity and displayed a pH-sensitive release pattern, with a sustained release profile of the IFS. PD/IFS nanoparticles exhibited remarkable in vitro anticancer activity comparable to that of free IFS solution in a concentration dependent manner in MG63 and Saos-2 cancer cells. PLGA-dextran by itself did not affect cell viability of cancer cells indicating its excellent biocompatibility. The formulation exhibited significantly higher PARP and caspase-3/7 expression in both the cancer cells.
\end{abstract}

Conclusion: Our study successfully demonstrated that nanoparticulate encapsulation of antitumor agent will increase the therapeutic efficacy and exhibit a greater induction of apoptosis and cell death.

Keywords: Ifosfamide, Osteosarcoma, Polymeric nanoparticles, Block copolymer, Apoptosis

\section{Background}

Osteosarcoma (OS) is one of the typical bone cancers that occur in distal femur and proximal tibia [1]. OS being mesenchymal in nature are very aggressive and more than $20 \%$ of cases are diagnosed at metastatic stage. Specifically, OS is commonly seen in children and adolescents [2]. Parallel to other solid tumors, OS tumors also contains a highly heterogeneous population of cancer cells in terms of growth rate, karyotype, antigenicity and chemosensitivity. Although 5-year survival rate increased to $65 \%$, yet it is way behind the overall cancer survival rate [3, 4]. Furthermore, survival rate of 5-year metastatic disease is still at a meager $20 \%$. At present, the therapies for OS treatment include surgical resection

\footnotetext{
*Correspondence: linxj1900123@gmail.com

${ }^{\dagger}$ Equal contributors

'Department of Orthopedic, The First Affiliated Hospital of Medical School of Zhejiang University, No. 79 Qingchun Road, Hangzhou, Zhejiang 310003,

China

Full list of author information is available at the end of the article
}

followed by chemotherapy regimens of various drugs including doxorubicin, cisplatin, and ifosfimide [5]. However, therapeutic activity of these drugs is limited by their severe drug-related toxicities such as cardiotoxicity and nephrotoxicity. Therefore, a therapeutic approach which is less toxic and highly effective in tumor is of utmost importance [6]. In this regard, present research is mainly focused on developing unique and novel therapeutic carriers to deliver the chemotherapeutic drugs to the cancer cells.

Ifosfamide (IFS) is a DNA-alkylating agent and a structural analog of cyclophosphamide. It acts as a prodrug, its metabolism occurring mainly through CYP 3A4 and CYP 2B6 enzymes, which are present predominantly in the hepatocytes $[7,8]$. IFS crosslinks DNA strands and inhibits DNA replication and ultimately leads to apoptosis due to activation of caspases in the cells. IFS is indicated as a mainline treatment for OS and delivered as an intravenous infusion [9]. A variety of nanoparticle- 
based delivery systems have been developed for the delivery of anticancer drugs. Self-assembled polymeric nanoparticles, have received increased attention for their potential application in biotechnology and medicine, especially as a drug delivery carrier in cancer therapeutics [10]. These amphiphilic nanoparticles usually have a hydrophobic core shielded by a hydrophilic shell when present in the aqueous environment. The hydrophobic core involves in the drug incorporation and the outer hydrophilic shell prevents the delivery system against reticuloendothelial system (RES) [11]. The polymeric selfassembled nanoparticles offer some unique advantages including core-shell morphology, high loading capacity, site-specific drug delivery, and avoids unwanted side effects of administered drug. Moreover, micelles remain stable in blood circulation for prolonged period of time and could avail enhanced permeability and retention effect (EPR) based passive targeting [12, 13].

Dextran, a polysaccharide is characterized as a colloidal and hydrophilic substance [14]. Dextran is extensively employed as a delivery carrier owing to its excellent biocompatible and immunoneutral properties. Moreover, hydroxyl group present in the glucose unit allow for easy chemical conjugations [15]. Biodegradable polymer, poly(lactic-co-glycolic acid) (PLGA) was selected due to its excellent systemic characteristics and biodegradability. Several studies have reported that nanosized PLGA NP would be in the ideal range of EPR effect as well as to avoid reticuloendothelial system (RES) mediated clearance. However, delivery characteristics of PLGA could be further improved by conjugating with hydrophilic dextran sulphate (DS) [16]. Recently, Jeong et al. reported that PLGA-dextran block copolymer forms self-assembling nanoparticles and could be used as a carrier to deliver multiple anticancer agents [17]. Consistently, we have synthesized a PLGA-dextran block copolymer via EDC/NHS chemistry and encapsulated IFS. We expected that incorporation of IFS in PLGA-dextran based polymeric nanoparticles will effectively increase the chemotherapeutic efficacy in cancers while at the same time reduce the overall side effects.

Thus far, the main aim of this study was to prepare ifosfamide-loaded PLGA-dextran polymeric nanoparticles for the treatment of osteosarcoma (OS). We hypothesized that IFS incorporation in a nanocarrier would increase its therapeutic effect due to the controlled release and defined properties. The dynamic light scattering analysis and morphology analysis were carried out to optimize the formulations. The biocompatible nature of blank nanoparticles (NP) and cytotoxic effect of IFSloaded NP was evaluated in MG63 and Saos-2 osteosarcoma cells via MTT assay. The apoptotic effect of free drug and IFS-loaded NP was studied means of PARP and caspase-3, which are typical apoptotic markers.

\section{Materials and methods Materials}

Ifosfamide ( $\geq 98 \%$ ) was purchased from Sigma Aldrich (St. Louis, MO, USA).Poly(d,l-lactic-co-glycolic acid) (PLGA) (Mw: 10,000; lactic acid : glycolic acid =50:50) was procured from Wako Pure Chemical (Tokyo, Japan). Dextran from Leuconostocspp was also obtained from Sigma-Aldrich (China). All other chemicals were reagent grade and used without further purifications.

\section{Synthesis of PLGA-Dextran block copolymer}

Approximately $3 \mathrm{~g}$ of PLGA-COOH was dissolved in anhydrous methylene chloride and to this organic solution, $70 \mathrm{mg}$ of NHS (N-hydroxysuccinimide) and $140 \mathrm{mg}$ of EDC (1-ethyl-3-(3-dimethylaminopropyl)-carbodiimide) was added. The organic mixture was stirred continuously for $12 \mathrm{~h}$ at room temperature. The $12 \mathrm{~h}$ time period is sufficient for the complete activation of carboxylic acid group in PLGA. The formed PLGA-NHS was precipitated by the addition of ice cold ether, washed with organic solvent mixture, and dried.

Aminated dextran was prepared as reported previously. Briefly, dextran and cyanoborohydride was mixed in a DMSO medium and to this mixture hexamethylene diamine was added and allowed the reaction for $24 \mathrm{~h}$. The amine group terminated dextran was collected, dialyzed, and lyophilized. To prepare the block copolymer, $100 \mathrm{mg}$ of PLGA-NHS and $125 \mathrm{mg}$ of dextran was dissolved in DMSO and inert atmosphere was maintained throughout the reaction time. The formed PLGA-dextran was dialyzed using dialysis membrane (molecular weight cutoff, $10,000 \mathrm{~g} / \mathrm{mol}$ ) for 3 days. The resulting products was lyophilized and dried under vacuum conditions.

\section{Preparation of Ifosfamide-loaded polymeric nanoparticles} IFS-loaded polymeric nanoparticles (NP) were prepared by precipitation method. In brief, $25 \mathrm{mg}$ of PLGAdextran (PLD) and $5 \mathrm{mg}$ of IFS were dissolved in $5 \mathrm{ml}$ of DMSO and to this mixture $20 \mathrm{ml}$ of ultra-pure water were added. The mixture was magnetic stirred for $2 \mathrm{~h}$ and followed by dialysis against distilled water. The dialysis process was continued for $3-4 \mathrm{~h}$ and the resulting drug-loaded polymeric NP was collected and lyophilized.

\section{Drug loading}

The loading efficiency and loading capacity was determined as follows. In brief, $10 \mathrm{mg}$ of lyophilized NP was dissolved in $5 \mathrm{ml}$ of DMSO and sonicated for $15 \mathrm{~min}$. The organic solution was centrifuged and the supernatant was used to calculate the amount of drug loaded. The drug loading was quantified using HPLC method. The HPLC system (Shimadzu, Kyoto, Japan) consisted of LC-10AT pump, a SPD-10A UV/Vis detector and a DGU-14A degasser model. The flow rate was maintained at $1 \mathrm{ml} / \mathrm{min}$. The 
wavelength of detection was $254 \mathrm{~nm} .50 \mathrm{mM}$ of $\mathrm{KH}_{2} \mathrm{PO}_{4}$ (pH 5.0) was used as a mobile phase.

\section{Particle size and size distribution analysis}

The average particle size and size distribution analysis was performed using a Zetasizer Nano-S90 (Malvern Instruments, Malvern, UK) and a $633 \mathrm{~nm} \mathrm{He-Ne} \mathrm{laser}$ beam at a fixed scattering angle of $90^{\circ}$. A dilute solution of NP was used to analyse the particle size. The experiments were performed in triplicates.

\section{Transmission electron microscopy}

The morphology of the PD/IFS was examined on a transmission electron microscope (JEOL JEM-200CX). Before the examinations, NP dispersion was diluted many times with ultra-pure water. The aqueous solution was dropped on the carbon coated copper grid and counter stained with $2 \%$ phosphotungistic acid. The samples were dried using an infrared lamp and viewed under TEM.

\section{Drug release study}

The IFS release from the PD/IFS NP system was determined using a dialysis method. Briefly, $30 \mathrm{mg}$ of PD/IFS lyophilized powder was dissolved in $1 \mathrm{ml}$ of water and sealed in a dialysis tube. The dialysis tube was in turn placed in a $50 \mathrm{ml}$ of Falcon tube containing $25 \mathrm{ml}$ of release media. Selective release media including phosphate buffered saline (PBS, pH 7.4) and acetate buffered saline (ABS, pH 5.5) was used. The main reason behind the selection of different $\mathrm{pH}$ was to mimic the conditions of tumor microenvironment. The sampling was done at specific time points such as $1,2,4.6,8,10,12,24,48,72,96,120 \mathrm{~h}$. At each sampling point, $1 \mathrm{ml}$ of release sample was withdrawn and replaced with equal volume of fresh media. The released IFS content in the released medium was determined by HPLC as previously described.

\section{Cell culture}

MG63 and Saos-2 osteosarcoma cancer cells were grown in DMEM supplemented with $10 \% \mathrm{FBS}, 100$ units/mL penicillin and $100 \mu \mathrm{g} / \mathrm{mL}$ of streptomycin. Cells were maintained at $37{ }^{\circ} \mathrm{C}$ with $5 \% \mathrm{CO} 2$ in a humidified incubator.

\section{Cell viability assay}

Cell viability was assessed using 3-(4,5-Dimethylthiazol-2yl)-2,5-diphenyltetrazolium bromide (MTT) calorimetric assay. MG63 and Saos-2 osteosarcoma cancer cells were seeded in a 96-well plate (4000 cells/well) and allowed to grow for 48-72 h. Next day, media was removed and replaced with fresh media containing blank PLGA-dextran, free IFS, and PD/IFS NP in a concentration-dependent manner. The formulations were incubated for $24 \mathrm{~h}$ and cell viability was estimated using MTT solution. MTT reagent $20 \mu \mathrm{L}$ in PBS was added into each well and the plate was incubated for $4 \mathrm{~h}$ at $37^{\circ} \mathrm{C}$. The culture medium in the wells was removed and $200 \mu \mathrm{L}$ of dimethylsulfoxide (DMSO) was added into the wells. The optical density of the solution was measured at $570 \mathrm{~nm}$ with a microplate reader. The mean value and standard deviation for each treatment were determined and then converted values relative to the control. IC50 were calculated using GraphPad Prism software.

\section{Morphological cell imaging}

Cover slips were rinsed in $70 \%$ ethanol for $10 \mathrm{~min}$ and washed with PBS. The cells were seeded into the cover slips and allowed to attach for $12 \mathrm{~h}$. The formulations as mentioned above was added to each well and further incubated for $24 \mathrm{~h}$. Then samples were washed with PBS, fixed with formalin (Sigma), and viewed under Nikon Eclipse 60i microscope system.

\section{Caspase-3 activity}

The activity of caspase- 3 was measured by colorimetric assay kits (Sigma-Aldrich) as per the manufacturer's protocols. MG63 and Saos-2 osteosarcoma cancer cells were seeded in a 6-well plate $\left(1 \times 10^{6}\right.$ cells/well $)$ and allowed to attach for $24 \mathrm{~h}$. Next day, media was removed and replaced with fresh media containing blank PLGA-dextran, free IFS, and PD/IFS NP in a concentration-dependent manner. The cells were incubated with respective formulations for $24 \mathrm{~h}$. Cell pellets were collected and treated with lysis buffer and incubated for $10 \mathrm{~min}$ in ice bath. The lysate was collected, centrifuged and supernatant was collected and evaluated for caspase- 3 activity.

\section{Apoptosis analysis}

FACS analysis is considered to be a specific and objective method for quantitative determination of apoptosis. MG63 and Saos- 2 cells were seeded at a density of $5 \times 10^{5}$ cells in a 6-well plate and incubated for $24 \mathrm{~h}$. When the cells reached $80 \%$ confluence, cells were treated with free IFS, and PD/IFS NP formulations $(1 \mu \mathrm{g} / \mathrm{ml})$ and further incubated for $24 \mathrm{~h}$. Following day, cells were harvested, washed, and incubated with a mixture of $0.25 \mathrm{mg} / \mathrm{mL}$ Annexin-V FITC and $10 \mathrm{mg} / \mathrm{mL}$ PI. The mixture was kept for $15 \mathrm{~min}$ at $37^{\circ} \mathrm{C}$. Excess PI and AV-FITC fluorescence were then washed off and cells were measured by flow cytometry (FACS Calibur, BD Biosciences). A minimum of 10,000 events was counted per sample by flow cytometry.

\section{Statistical analysis}

Results in the present study are presented as means \pm standard deviations. Statistical significance was evaluated by analysis of variance (ANOVA), followed by Tukey's post-hoc test. "P-values of $p<0.05$ was considered to be statistically significant. 


\section{Results}

\section{Characterization of PD/IFS nanoparticles}

PLGA-dextran formed self-assembled polymeric micelles in the aqueous medium. Generally, PLGA is hydrophobic, so it should form the inner core of the polymeric micelle while the dextran domain should form the outer shell due to its hydrophilic nature [18]. Polymeric micelles incorporated drugs by the hydrophobic interaction between the drug and the hydrophobic domain of the block copolymer. It has been frequently reported that polymeric micelles enhances accumulation in tumor cells and prolongs blood circulation times [19].

\section{Particle size analysis}

The particle size and size distribution of PD/IFS NP was investigated by means of dynamic light scattering (DLS) technique. The particle size of PD/IFS was observed to be $124 \pm 3.45 \mathrm{~nm}$ with an excellent dispersity index of 0.124 (PDI) (Fig. 1a). Blank polymeric micelles posted an average size of $75 \pm 2.39 \mathrm{~nm}$. The increase in particle size upon drug incorporation might due to the bulkier core of micellar system. Furthermore, it has been frequently reported that small particle size $<200 \mathrm{~nm}$ could accumulate preferentially in the tumor tissues via enhanced permeability and retention (EPR) effect. Other than this, small particle size could effectively evade the RES based clearance system in the blood circulation [19].

\section{Morphological analysis}

The particle size of PD/IFS NP was further confirmed by TEM imaging. As seen from Fig. 1b, particle sizes were in the range of $60-80 \mathrm{~nm}$ and uniformly distributed in the carbon coated copper grid. The particles were clearly spherical and present as a dense black object the TEM grid. No apparent sign of aggregation was seen among the particles. It has to be noted that particle size observed form TEM was smaller than observed from DLS analysis. The difference in particle size might be attributed to the dried state (from TEM) and hydrated state (from DLS) of particles.

\section{Drug loading and In vitro drug release}

IFS was effectively entrapped in the NPs with a loading and encapsulation efficiency of $20.15 \pm 3.5 \%$ and $89 \pm$ $1.95 \%$, respectively. The release profile of IFS from PD/ IFS NP was performed in phosphate buffered saline (PBS) and acetate buffered Saline (ABS) at $37^{\circ} \mathrm{C}$. Results showed that IFS released in a sustained manner throughout the study period up to $96 \mathrm{~h}$ (Fig. 2). As expected, PD/IFS showed a $\mathrm{pH}$-dependent release profile with accelerated release in the acidic $\mathrm{pH}$ than comparing to that of physiological $\mathrm{pH}$ conditions. It has to be noted that accelerated release of drug from the NP might be attributed to the fast diffusion of drug and partially due to the higher degradation of delivery vehicle in the acidic conditions. Broadly, release profile of IFS in pH 7.4 and pH 5.0 could be divided into two parts; first, faster release of IFS was observed until $24 \mathrm{~h}$ and second, a relatively more sustained release phenomenon was observed from 24 to $96 \mathrm{~h}$ study period. For example, nearly $\sim 30 \%$ of IFS released in first $24 \mathrm{~h}$ while only $\sim 55 \%$ of drug released by the end of $96 \mathrm{~h}$ in PBS media. Similar trend was observed in ABS media, where nearly $\sim 40 \%$ of drug released in $24 \mathrm{~h}$ and completed the release $(100 \%)$ by the end of $96 \mathrm{~h}$. The sustained release of drug in $\mathrm{pH} 7.4$ condition and accelerated release in $\mathrm{pH} 5.0$ conditions would be advantageous in cancer drug delivery.

\section{Cytotoxicity assay and cellular morphology}

The cancer cells were treated with blank NP with different concentrations ranging from 0.1 to $100 \mu \mathrm{g} / \mathrm{mL}$ (Fig. 3a, b). The results clearly showed that synthesized polymers were highly biocompatible and showed a cell viability of more than $90 \%$ throughout all the concentrations tested. Fluorescent images of MG63 cells showed that cells maintained their morphology when incubated with blank NP (Fig. 3c). The polymeric carrier itself did not contribute to cytotoxicity is very advantageous.

Cytotoxic potential of free IFS and PD/IFS was evaluated in both the osteosarcoma cancer cell lines. The cells were cultured in the presence of free IFS and PD/IFS NP at increasing concentrations of drugs. As shown in
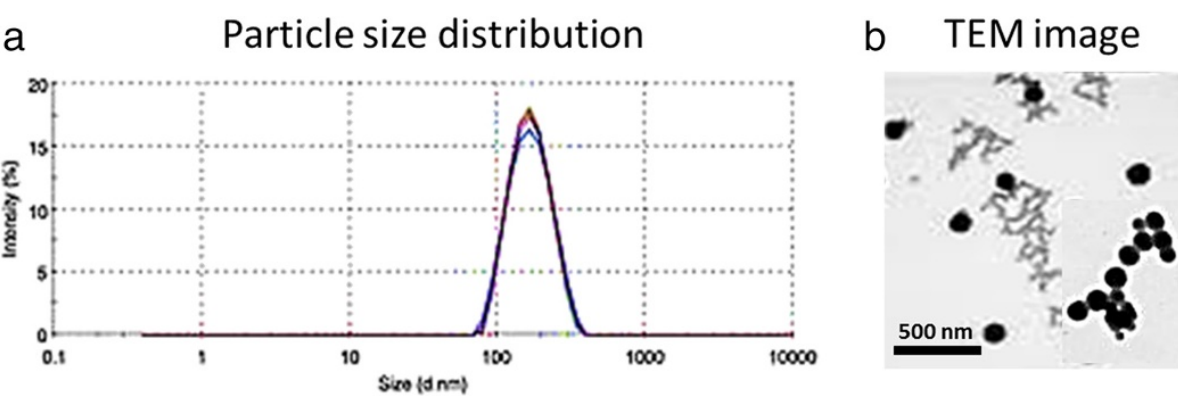

Fig. 1 a Particle size distribution of ifosfamide-loaded PLGA-dextran (PD/IFS) nanoparticles $\mathbf{b}$ TEM image of PD/IFS 


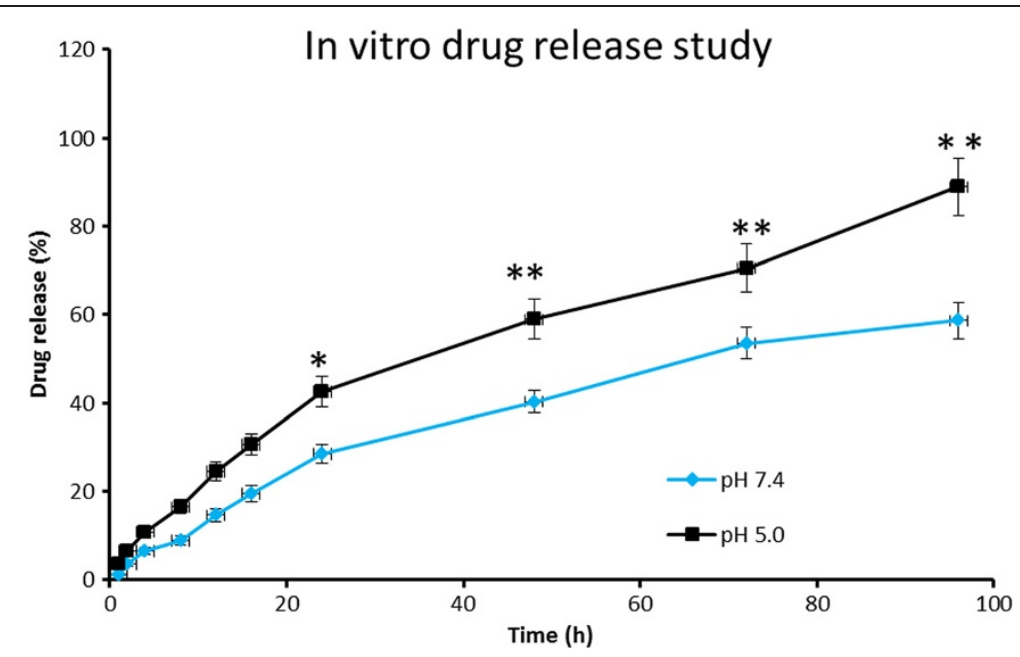

Fig. 2 The release profile of IFS from PLGA-dextran nanoparticulate system. The release study was performed in phosphate buffered saline and acetate buffered saline. The study was carried out for $96 h .{ }^{* *} p<0.01$ is the statistical difference between pH 7.4 and pH 5.5 release medium

Fig. 4a, b, both free IFS and PD/IFS were able to effectively inhibit cell growth and showed a concentration dependent-cytotoxic effect.

Furthermore, morphology of cells treated with free IFS and PD/IFS NP was evaluated by optical microscope. In both the case, untreated cells presented a well-defined morphology and adhered to the cover slip in the 6-well plate (Fig. 4c, d). In case of PD/IFS treated group, marked presence of dead cells were observed. The cells were either fusiform or rounded and in the process of dying indicating the cytotoxic effect of the optimized formulations.
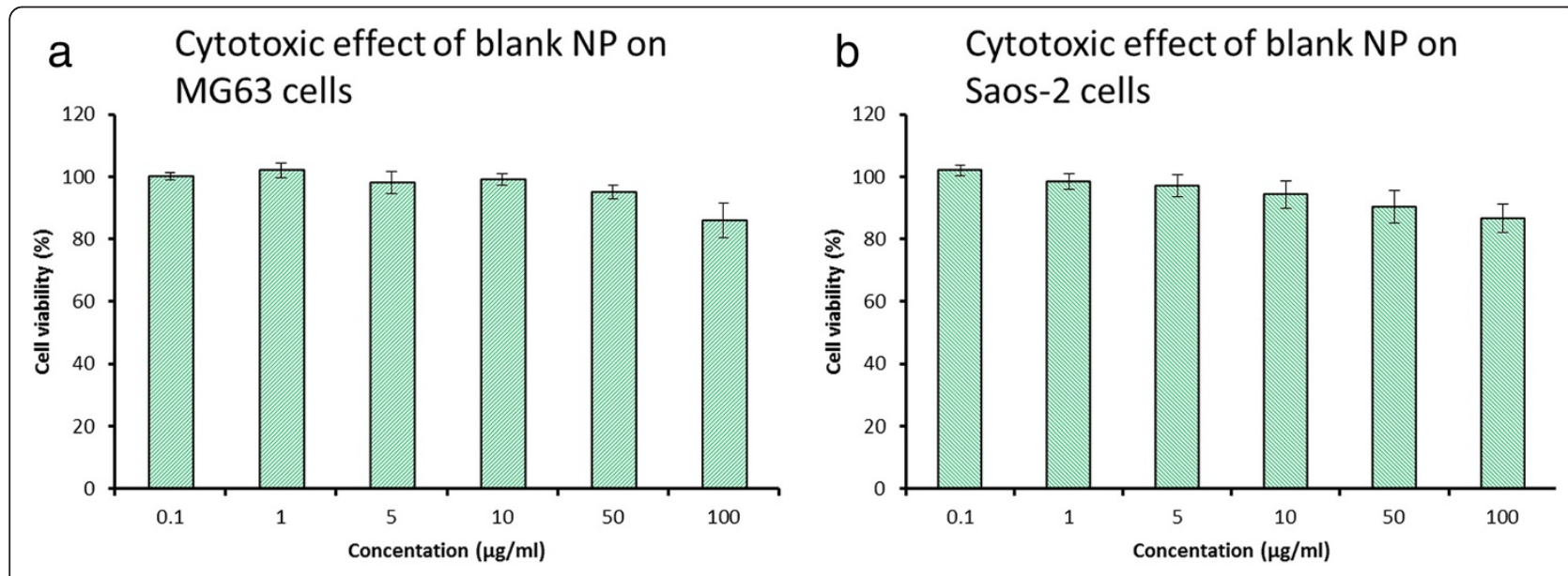

C

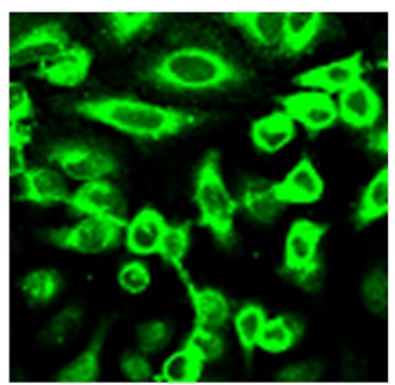

Fig. 3 Cytotoxicity assay for blank nanoparticles in a MG63 b Saos-2 osteosarcoma cancer cells. c Confocal laser scanning microscopic images of PD/IFS 

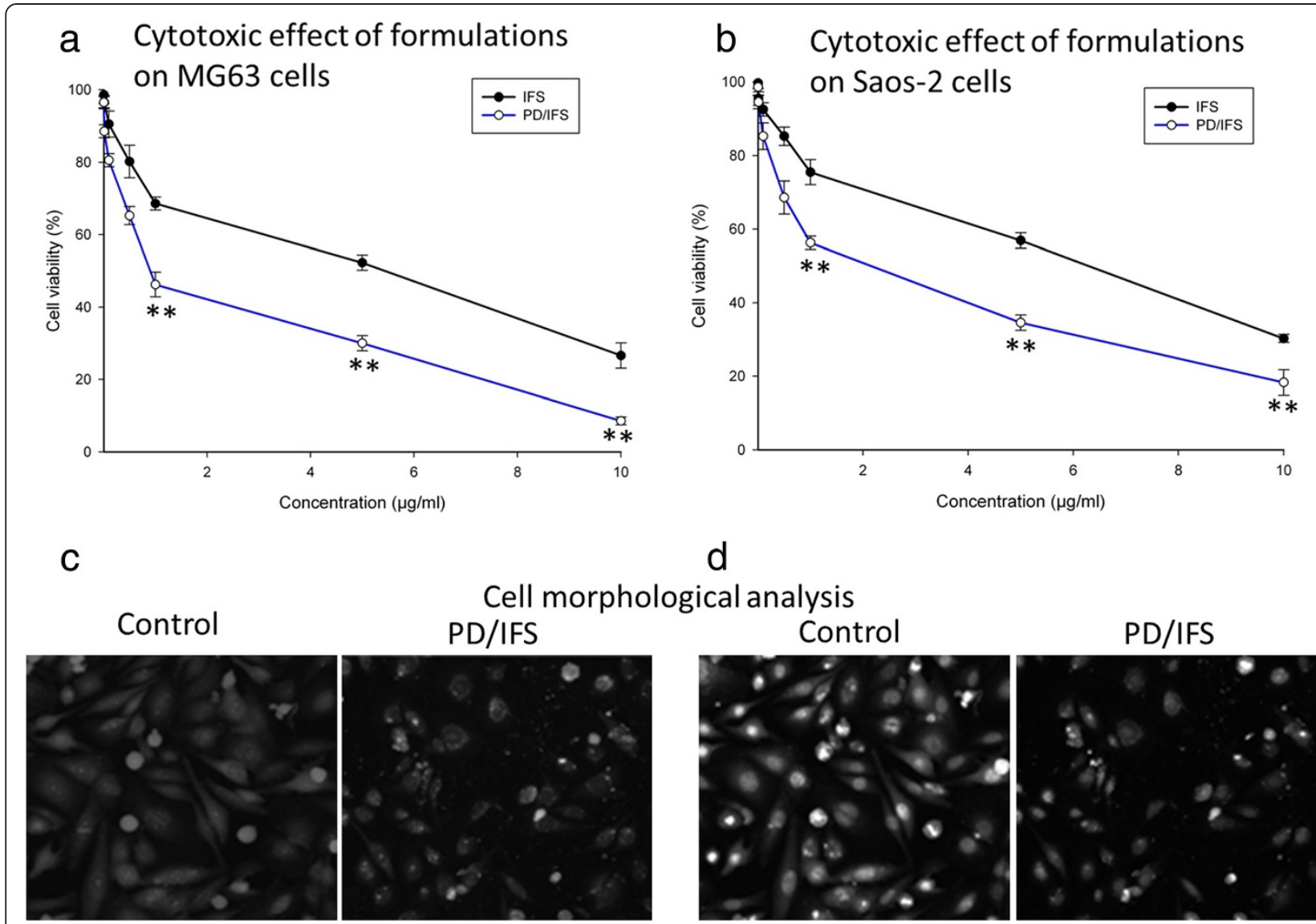

d

Fig. 4 Cytotoxicity of free IFS and PD/IFS (with equivalent IFS concentration) on (a) MG63 (b) Saos-2 osteosarcoma cancer cells. The cytotoxicity assay was performed by MTT technique. The cells were incubated for $24 \mathrm{~h}$ and the experiment was repeated four times in triplicate. Optical images of (c) MG63 (d) Saos-2 cells after incubation with free IFS and PD/IFS for $24 \mathrm{~h} .{ }^{* *} p<0.01$ is the statistical difference between IFS and PD/ IFS in both cancer cells

\section{Cellular apoptosis analysis}

The ability free IFS and PD/IFS NP to induce apoptosis on representative MG63 and Saos-2 cancer cell was evaluated by means of Annexin-V/PI-mediated apoptosis analysis. It can be clearly seen (Fig. 5a, b) that PD/IFS induced a greater apoptosis rate in both the cancer cells. Caspase- 3 activity was analysed in the cancer cells to further prove the apoptosis behaviour of respective formulations (Fig. 6a, b). Consistent with apoptosis analysis, PD/IFS showed a significantly $(p<0.01)$ higher expression of caspase 3 in MG63 cancer cells in a concentration dependent manner. Similar trends were observed in Saos-2 cancer cells however, caspase-3 level was relatively than expressed in MG63 cells.

\section{Discussion}

Osteosarcoma (OS) is one of the typical bone cancers that occur in distal femur and proximal tibia. Although technological advancement increased the 5-year survival rate to $65 \%$, yet it is way behind the overall cancer survival rate. Furthermore, the metastatic or recurring disease 5-year survival rate is still at a meager $20 \%$. At present, the therapies for OS treatment include surgical resection followed by chemotherapy regimens of various drugs including doxorubicin, cisplatin, and ifosfamide. Specifically, IFS, a DNA-alkylating agent is indicated as a mainline treatment for OS. IFS crosslinks DNA strands and inhibits DNA replication and ultimately leads to apoptosis due to activation of caspases in the cells. In order to increase its therapeutic efficacy, it has to be loaded in nanoparticle-based delivery systems. A selfassembled polymeric nanoparticle which has a hydrophobic core, involves in the drug incorporation and the outer hydrophilic shell prevents the delivery system against reticuloendothelial system (RES). In this study, PLGA-dextran copolymer was synthesized and used to encapsulate IFS. Biodegradable polymer, poly(lactic-coglycolic acid) (PLGA) was selected due to its excellent systemic characteristics and biodegradability. Dextran was selected due to its hydrophilic nature and biocompatibility. Dextran has an advantage, in that it has a confluent functional (hydroxyl) group in its chain, and the hydroxyl 


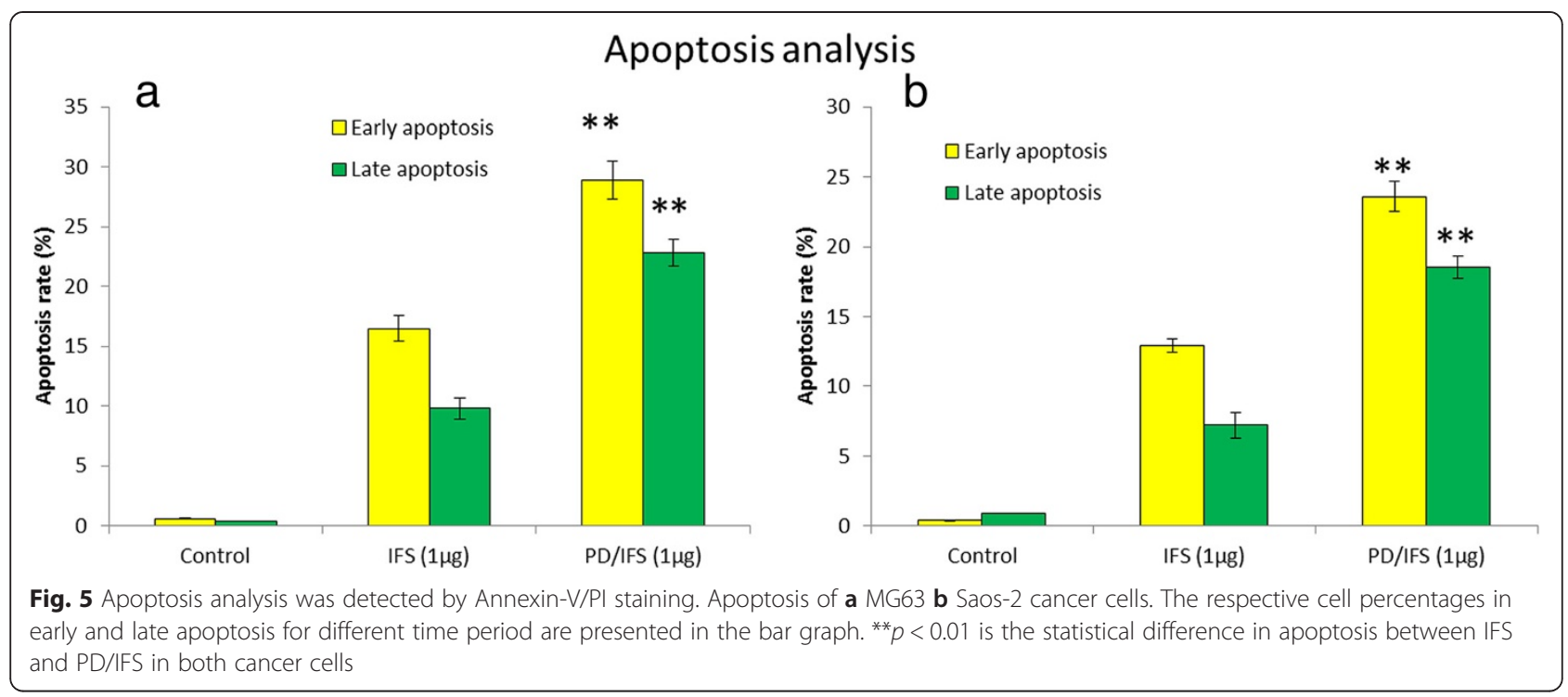

group can be used for chemical modification with targeting moieties. To conjugate PLGA copolymer, $-\mathrm{COOH}$ group of PLGA was activated by means of NHS to form PLGA-NHS. This PLGA-NHS was then mixed with aminated dextran to form block copolymer (Fig. 7). We expected that incorporation of IFS in PLGA-dextran based polymeric nanoparticles will effectively increase the chemotherapeutic efficacy in cancers while at the same time reduce the overall side effects.

One of the most important criteria for successful cancer targeting is the development of a biocompatible and safe nanoparticulate system. The biocompatibility of PLGA-dextran blank NP was studied in MG63 and Saos-2 osteosarcoma cancer cells. The results clearly showed that synthesized polymers were highly biocompatible and showed a cell viability of more than $90 \%$ throughout all the concentrations tested.

Cytotoxic potential of free IFS and PD/IFS was evaluated in both the osteosarcoma cancer cell lines. Throughout all the concentrations, PD/IFS showed significant anticancer effect than comparing to free IFS. IC50 values of free IFS and PD/IFS NP were determined to quantify the cytotoxic effect. IC50 value of free IFS and PD/IFS NP were $5.24 \mu \mathrm{g} / \mathrm{ml}$ and $0.932 \mu \mathrm{g} / \mathrm{ml}$, respectively in MG63 cancer cells, whereas, it was $5.46 \mu \mathrm{g} / \mathrm{ml}$ and $1.046 \mu \mathrm{g} / \mathrm{ml}$, respectively in Saos-2 cancer cells. It should be mentioned that PLGA-dextran alone did not affect cell viability. Therefore, the therapeutic efficacy is only due to the drug loaded within the nanoparticles. The nanoparticle adsorbed onto the cell membrane which resulted in an increase in the intracellular drug concentration, offering a

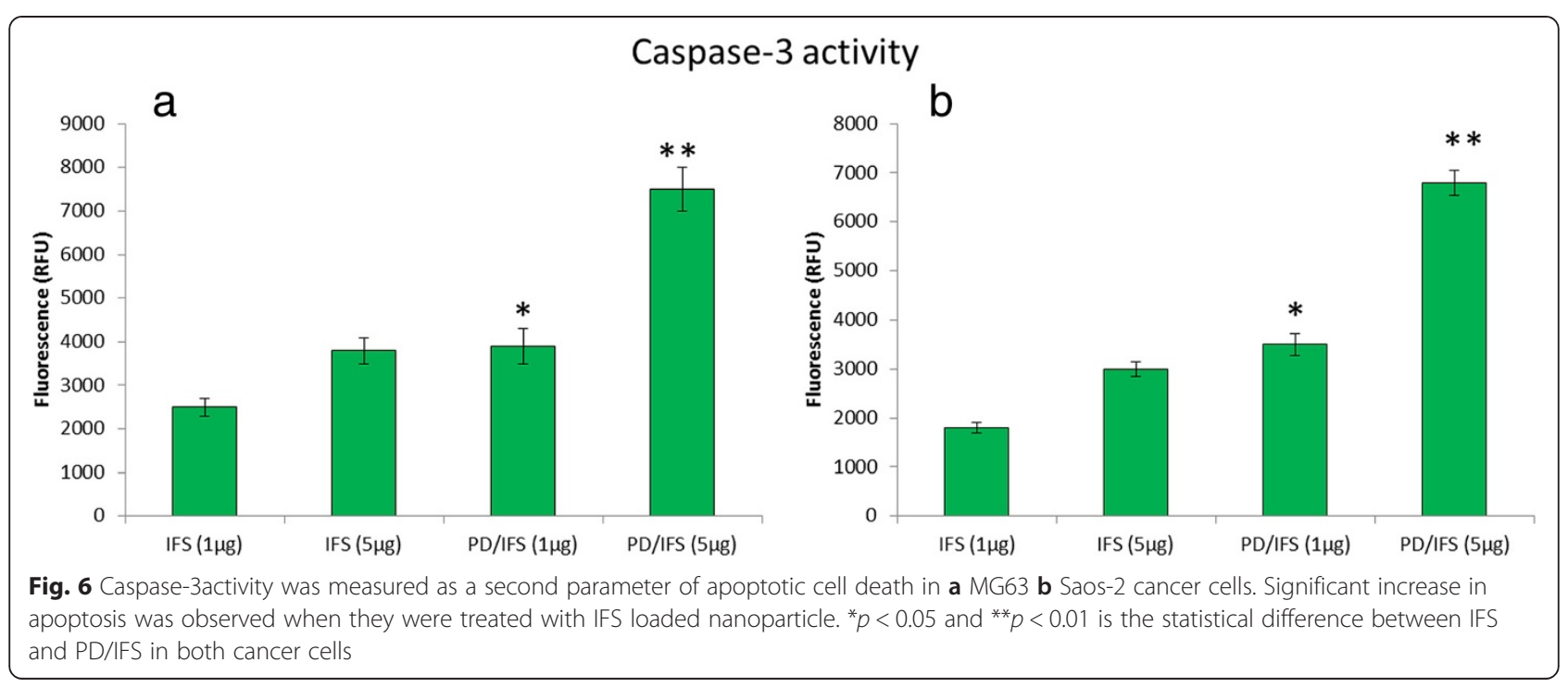




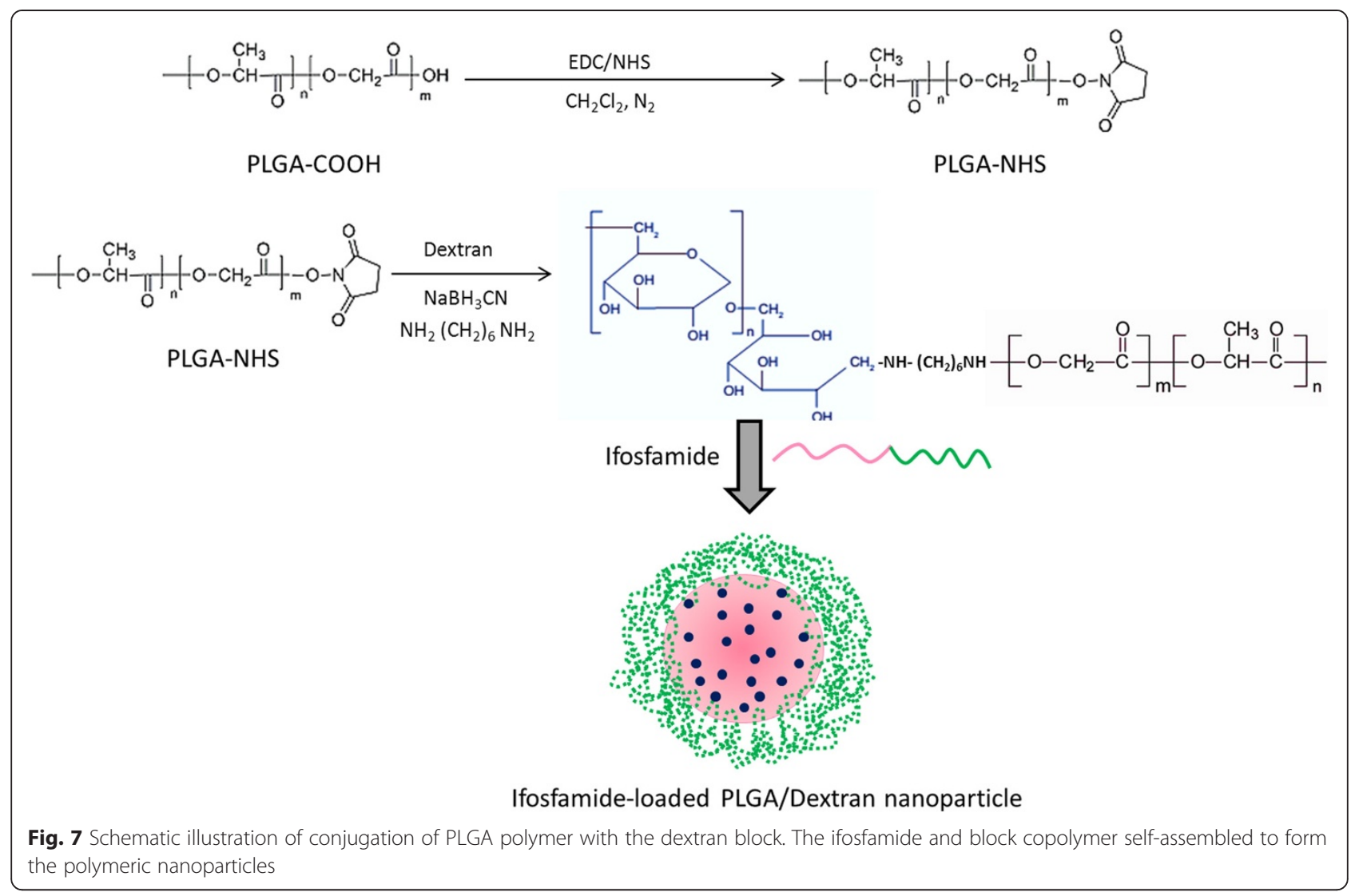

gradient that would favour drug influx into the cells [20]. Moreover, efficient uptake of NP could be a potential contributing factor in the enhanced cytotoxic effect of delivery systems [21].

Consistent with cytotoxicity assay, PD/IFS showed a significantly $(p<0.01)$ higher apoptosis of cancer cells in MG63 cancer cells. Similar trends were observed in Saos-2 cancer cells however, apoptosis rate was relatively than MG63 cells. The difference in apoptosis rate between two cell lines could be due to the biological origin and its growth rate. Consistent with cell apoptosis, PD/ IFS showed a significantly $(p<0.01)$ higher expression of caspase 3/7 in MG63 cancer cells in a concentration dependent manner. Similar trends were observed in Saos-2 cancer cells however, caspase-3 level was relatively than expressed in MG63 cells. Therefore it is clear that nanoparticulate formulation of IFS remarkably increased the therapeutic performance of anticancer drug.

In the clinical setting, anticancer drugs often lead to systemic toxicity which restricts the overall dose. A limited dose however will limit the therapeutic index of given anticancer drugs. This essentially promotes the phenomenon of multi drug resistance (MDR) in cancer cells which will further complicate the drug treatment. In this regard, EUROMOS trail shows that free drug
(IFS) does not improve the post-operative chemotherapy and patients responded poorly. In addition, IFS increased the number of side effects in various patients. Therefore, we believe the incorporation of IFS in a nanoparticulate system could potentially improve its therapeutic efficacy while at the same time is expected to reduce its side effects. Our study successfully demonstrated that nanoparticulate encapsulation of antitumor agent will increase the therapeutic efficacy and exhibit a greater induction of apoptosis and cell death. It seems that the nanoparticle delivery system caused increased uptake of IFS and distribution in the nucleus resulting in the enhanced cell death [22]. Our study is consistent with previously published report that nanoparticle-drug conjugates induce stronger activation of apoptosis signalling pathways comparing to that of free drug. A thorough study on experimental animal models and different cell panel would bring more value to the osteosarcoma treatment.

\section{Conclusion}

Ifosfamide-loaded PLGA-dextran polymeric nanoparticles (PD/IFS) were successfully developed and studied its anticancer efficacy against multiple osteosarcoma cancer cells. The drug-loaded nanoparticle was characterized in terms 
of size distribution, morphology, zeta potential, drug loading, release profile, cytotoxicity assay, and apoptosis induction. The formulated PD/IFS showed a high drug loading capacity and displayed a $\mathrm{pH}$-sensitive release pattern, with a sustained release profile of the IFS. This property is important for all the biomedical applications including cancer chemotherapy. PD/IFS nanoparticles exhibited remarkable in vitro anticancer activity comparable to that of free IFS solution in a concentration dependent manner in MG63 and Saos-2 cancer cells. PLGA-dextran by itself did not affect cell viability of cancer cells indicating its excellent biocompatibility. The formulation exhibited significantly higher PARP and caspase- 3 expression in both the cancer cells. Our study successfully demonstrated that nanoparticulate encapsulation of antitumor agent will increase the therapeutic efficacy and exhibit a greater induction of apoptosis and cell death. Thus, IFS-loaded PLGA-dextran based formulations could be a potential candidate for the treatment of osteosarcoma.

\section{Competing interest}

The authors report no conflict of interest.

\section{Author's contributions}

BC and JZY carried out the main experimental parameters. LFW and YJZ have carried out the cell-based assays and protocols. XJL has designed and written the entire manuscript. All the authors of this paper read and approved the final version of this manuscript.

\section{Acknowledgements}

The financial assistance to the authors of this work was covered under the 'Research Fellowship' from 'Council of Medical Research' of Zhejiang University, China. One of the authors acknowledges the support of Dr. Ji Han Yang during the course of work.

\section{Author details}

'Department of Orthopedic, The First Affiliated Hospital of Medical School of Zhejiang University, No. 79 Qingchun Road, Hangzhou, Zhejiang 310003, China. ${ }^{2}$ Department of Laboratoire Central, The First Affiliated Hospital of Medical School of Zhejiang University, Hangzhou 310003, China.

Received: 7 April 2015 Accepted: 8 October 2015

Published online: 21 October 2015

\section{References}

1. Fletcher C, Unni K, Mertens F. Pathology and genetics of tumours of soft tissue and bone. Lyon: IARC Press; 2002.

2. Schwartz CL, Gorlick R, Teot L, Krailo M, Chen Z, Goorin A, et al. Children's Oncology Group: multiple drug resistance in osteogenicsarcoma: INT0133 from the Children's Oncology Group. J ClinOncol. 2007;25:2057-62.

3. Chou AJ, Gorlick R. Chemotherapy resistance in osteosarcoma: current challenges and future directions. Expert Rev Anticancer Ther. 2006;6:1075-85.

4. Kufe DW, Pollock RE, Weichselbaum RR, Bast RC, Gansler TS, Holland JF, et al. Holland-Frei Cancer Medicine, 6th ed., Hamilton (ON):BC Decker; 2003.

5. Luetke A, Meyers PA, Lewis I, Juergens H. Osteosarcoma treatment - where do we stand? A state of the art review. Cancer Treat Rev. 2014:40:523-32.

6. PosthumaDeBoer J, van Royen B, Helder M. Mechanisms of therapy resistance in osteosarcoma: a review. Oncol Discovery. 2013;1:8-15.

7. Kumar MK, Shah MH, Ketkar A, et al. Effect of drug solubility and different excipients on floating behaviour and release from glyceryl monooleate matrices. Int J Pharm. 2004;272:151-60.

8. Trickler WJ, Nagvekar AA, Dash AK. A novel nanoparticle formulation for sustained paclitaxel delivery. AAPS PharmSciTech. 2008:9:486-93.

9. Zhang J, Tian Q, Zhou S. Clinical pharmacology of cyclophosphamide and ifosfamide. Curr Drug Ther. 2006;1:55-84.
10. Page $\mathrm{R}$, Takimoto $\mathrm{C}$. Cancer management: a multidisciplinary approach: medical, surgical, and radiation oncology. In: Pazdur R, editor. Principles of chemotherapy8th edition. New York: PRP; 2004. p. 21-38.

11. Sahoo SK, Labhasetwar V. Enhanced antiproliferative activity of transferrinconjugated paclitaxel-loaded nanoparticles is mediated via sustained intracellular drug retention. Mol Pharm. 2005;2:373-83.

12. Jeong YI, Jin SG, Kim IY, Pei J, Wen M, Jung TY, et al. Doxorubicinincorporated nanoparticles composed of poly(ethylene glycol)-grafted carboxymethyl chitosan and antitumor activity against glioma cells in vitro. Colloids Surf B Biointerfaces. 2010;79:149-55.

13. Maeda H, Wu J, Sawa T, Matsumura Y, Hori K. Tumor vascular permeability and the EPR effect in macromolecular therapeutics: a review. J Control Release. 2000;65:271-84.

14. Du YZ, Weng Q, Yuan $H$, et al. Synthesis and antitumor activity of stearateg-dextran micelles for intracellular doxorubicin delivery. ACS Nano. 2010;4:6894-902.

15. Bisht S, Maitra A. Dextran-doxorubicin/chitosan nanoparticles for solid tumor therapy. Wiley Interdiscip Rev NanomedNanobiotechnol. 2009;1:415-25.

16. Matsumoto A, Matsukawa $Y$, Horikiri $Y$, Suzuki T. Rupture and drug release characteristics of multi-reservoir type microspheres with poly(dl-lactide-coglycolide) and poly(dl-lactide). Int J Pharm. 2006;327:110-6.

17. Jeong Yl, Choi KC, Song CE. Doxorubicin release from core-shell type nanoparticles of poly(DL-lactide-co-glycolide)-grafted dextran. Arch Pharm Res. 2006:29:712-9.

18. Kwon GS, Naito M, Yokoyama M, et al. Physical entrapment of adriamycin in AB block copolymer micelles. Pharm Res. 1995;12:192-5.

19. Yang $R$, Shim WS, Cui FD, et al. Enhanced electrostatic interaction between chitosan-modified PLGA nanoparticle and tumor. Int J Pharm. 2009;371:142-7.

20. Leroux JC, Doelker E, Gurny R. The use of drug-loaded nanoparticles in cancer chemotherapy. In: Benita S, editor. Microencapsulation: methods and industrial applications. New York: Marcel Dekker; 1996. p. 535-75.

21. Santos JL, Oliveira H, Pandita D, Rodrigues J, Pêgo AP, Granja PL, et al. Functionalization of poly(amidoamine) dendrimers with hydrophobic chains for improved gene delivery in mesenchymal stem cells. J Control Release. 2010;144:55-64.

22. Chen BA, Dai YY, Wang XM, Zhang RY, Xu WL, Shen HL, et al. Synergistic effect of the combination of nanoparticulate $\mathrm{Fe} 3 \mathrm{O} 4$ and $\mathrm{Au}$ with daunomycin on K562/A02 cells. Int J Nanomedicine. 2008;3:343-50.

\section{Submit your next manuscript to BioMed Central and take full advantage of:}

- Convenient online submission

- Thorough peer review

- No space constraints or color figure charges

- Immediate publication on acceptance

- Inclusion in PubMed, CAS, Scopus and Google Scholar

- Research which is freely available for redistribution 